\title{
LOCAL BOUNDEDNESS AND CONTINUITY FOR A FUNCTIONAL EQUATION ON TOPOLOGICAL SPACES ${ }^{1}$
}

\author{
C. T. NG
}

\begin{abstract}
It is known that the locally bounded solutions $f$ of Cauchy's functional equation $f(x)+f(y)=f(x+y)$ on the reals are necessarily continuous. We shall extend this result to the functional equation $f(x)+g(y)=h(T(x, y))$ on topological spaces.
\end{abstract}

1. Introduction. Let $X, Y$ be topological spaces and let $f: X \rightarrow R$ (the reals), $g: Y \rightarrow R, T: X \times Y \rightarrow R$ and $h: T(X \times Y) \rightarrow R$ be functions satisfying the functional equation

$$
f(x)+g(y)=h(T(x, y))
$$

for all $x \in X, y \in Y$. We shall give some sufficient topological assumptions on $X$ and $T$ so that the local boundedness and nonconstancy of $f$ insure that $g$ is continuous. The method was suggested by the work of J. Pfanzagl in his paper [6] generalizing a result of G. Darboux [2].

\section{Main theorems.}

THEOREM 1. For equation (1), if each pair of points of $X$ is contained in the continuous image of some connected and locally connected space (for instance, when $X$ is connected and locally connected or when $X$ is pathwise connected), $T$ is continuous in each of its two variables and $f$ is nonconstant and locally bounded from above (or from below) at each point of $X$, then $g$ is continuous on $Y$.

Proof. Let $a, b \in X$ be such that $f(a) \neq f(b)$. There exist a connected and locally connected space $\tilde{X}$ and a continuous mapping $\gamma: \tilde{X} \rightarrow X$ such that $a, b \in \gamma(\tilde{X})$. The functions $\tilde{f}:=f \circ \gamma$ and $\tilde{T}$ with $\tilde{T}(\tilde{x}, y):=T(\gamma(\tilde{x}), y)$ for $\tilde{x} \in \tilde{X}, y \in Y$, now satisfy the induced functional equation

$$
\tilde{f}(\tilde{x})+g(y)=h(\tilde{T}(\tilde{x}, y))
$$

Received by the editors October 27, 1972.

AMS (MOS) subject classifications (1970). Primary 39A15, 39A20, 39A40; Secondary 54C30, 54D05.

Key words and phrases. Functional equations, local boundedness, connected, locally connected, continuous.

${ }^{1}$ Research supported under Canadian NRC Grant A8212.

(c) American Mathematical Society 1973 
for all $\tilde{x} \in \tilde{X}, y \in Y$. The local boundedness of $f$ passes to $\tilde{f}$ and the continuity of $T$ in each variable passes to $\tilde{T}$. With this observation there is no loss of generality if we suppose from the very beginning that $X$ is connected and locally connected.

Since $X$ is connected and $f$ is nonconstant on $X, f$ cannot be locally constant on $X$ and there exists a point $e \in X$ such that $f$ is nonconstant on every neighbourhood of $e$. As $X$ is locally connected and $f$ is locally bounded from above at $e$ there exists an open connected neighbourhood $U$ of $e$ on which $f$ is bounded from above. Thus $f$ is nonconstant and bounded from above on the connected and locally connected set $U$.

Let $x_{1}, x_{2} \in U$ be such that $f\left(x_{1}\right) \neq f\left(x_{2}\right)$. It follows from equation (1) that $T\left(x_{1}, y\right) \neq T\left(x_{2}, y\right)$ for all $y \in Y$.

Let $y_{0} \in Y$ be arbitrarily given and we shall prove the continuity of $g$ at $y_{0}$. We may suppose that $t_{1}:=T\left(x_{1}, y_{0}\right)<T\left(x_{2}, y_{0}\right)=: t_{2}$. By Lemma 1 in Pfanzagl [5] there exists a connected $B \subseteq U$ such that $\left.T\left(B, y_{0}\right)=\right] t_{1}, t_{2}[$. Let $\varepsilon>0$ be arbitrarily given. Since $\sup f(B)<\infty$, there exists $x_{0} \in B$ such that $f\left(x_{0}\right) \geqq f(x)-\varepsilon$ for all $x \in B$.

Let $M:=\left\{y \in Y: t_{1}<T\left(x_{0}, y\right)<t_{2}\right\}$. Then $y_{0} \in M$ and, as $T\left(x_{0}, \cdot\right)$ is continuous on $Y, M$ is a neighbourhood of $y_{0}$.

For each $\left.y \in M, T\left(x_{0}, y\right) \in\right] t_{1}, t_{2}\left[=T\left(B, y_{0}\right)\right.$ and so there exists $x \in B$ such that $T\left(x_{0}, y\right)=T\left(x, y_{0}\right)$. Thus $f\left(x_{0}\right)+g(y)=h\left(T\left(x_{0}, y\right)\right)=h\left(T\left(x, y_{0}\right)\right)=$ $f(x)+g\left(y_{0}\right)$. As $f\left(x_{0}\right) \geqq f(x)-\varepsilon$ we have $g(y) \leqq g\left(y_{0}\right)+\varepsilon$.

Let $x_{3}, x_{4} \in B$ be arbitrarily chosen such that $t_{3}:=T\left(x_{3}, y_{0}\right)<T\left(x_{0}, y_{0}\right)<$ $T\left(x_{4}, y_{0}\right)=: t_{4}$.

Let $N:=\left\{y \in Y: T\left(x_{3}, y\right)<T\left(x_{0}, y_{0}\right)<T\left(x_{4}, y\right)\right\}$. Then $y_{0} \in N$ and, as $T\left(x_{3}, \cdot\right)$ and $T\left(x_{4}, \cdot\right)$ are continuous on $Y, N$ is a neighbourhood of $y_{0}$.

For each $y \in N, T(B, y)$ is an interval of $R$ as $B$ is connected and $T(\cdot, y)$ is continuous. Furthermore, $T\left(x_{3}, y\right)$ and $T\left(x_{4}, y\right)$ are points of $T(B, y)$ with $T\left(x_{3}, y\right)<T\left(x_{0}, y_{0}\right)<T\left(x_{4}, y\right)$ and so $T\left(x_{0}, y_{0}\right) \in T(B, y)$. Hence there exists $x \in B$ such that $T\left(x_{0}, y_{0}\right)=T(x, y)$. From this we have $f\left(x_{0}\right)+g\left(y_{0}\right)=f(x)+g(y)$. As $f\left(x_{0}\right) \geqq f(x)-\varepsilon$ we have $g\left(y_{0}\right)-\varepsilon \leqq g(y)$.

$M \cap N$ is then a neighbourhood of $y_{0}$ and $g\left(y_{0}\right)-\varepsilon \leqq g(y) \leqq g\left(y_{0}\right)+\varepsilon$ for every $y \in M \cap N$. This proves the continuity of $g$ at $y_{0}$.

Remark 1. Lemma 1 in Pfanzagl [5] is given as: let $X$ be a connected and locally connected Hausdorff space, $\theta: X \rightarrow R$ a continuous map, then to any $t_{1}, t_{2} \in \theta(X)$ with $t_{1}<t_{2}$, there exists a connected component $B$ of $\theta^{-1}(] t_{1}, t_{2}[)$ such that $\left.\theta(B)=\right] t_{1}, t_{2}[$. The proof is based on a theorem of Wilder [7, p. 46, Theorem 3.8]. The assumption that $X$ is Hausdorff is however not used and can be removed.

COROLlary 1. If $X$ is locally connected, $T: X \times X \rightarrow R$ is continuous in each variable, $f: X \rightarrow R$ is locally bounded from above (or from below) at 
each point of $X$ and $h$ is any function on $T(X, X)$ satisfying the functional equation

$$
f(x)+f(y)=h(T(x, y))
$$

for all $x, y \in X$, then $f$ must be continuous on $X$.

Proof. For a point $a \in X$, if $f$ is locally constant at $a$ then $f$ is continuous at $a$. We may suppose now $f$ is not locally constant at $a$ and hence there exists an open connected neighbourhood $U$ of $a$ such that $f$ is bounded and nonconstant on $U$. We can apply Theorem 1 to the equation

$$
f(x)+f(y)=h(T(x, y))
$$

for all $x \in U, y \in X$ yielding the continuity of $f$ on $X$.

REMARK 2. Corollary 1 is proved by Pfanzagl [6] under stronger assumptions on $X$-that $X$ is locally compact and locally connected Hausdorff.

THEOREM 2. For equation (1), if each pair of points of $X$ is contained in some compact connected subset of $X, T$ is jointly continuous on the product space $X \times Y$ and $f$ is nonconstant and locally bounded from above on $X$ (or locally bounded from below on $X$ ), then $g$ is continuous on $Y$.

Proof. Similar to the argument given in the first paragraph in the proof of Theorem 1 we may suppose that $X$ is compact and connected. We note that $f$ is then bounded from above on every subset of $X$.

Let $y_{0} \in Y$ and $\varepsilon>0$ be arbitrarily given.

Since $f$ is nonconstant on $X$, for each $y \in Y$ the function $T(\cdot, y)$ is nonconstant on $X$ and $T(X, y)$ is a proper closed interval of $R$. Write $T\left(X, y_{0}\right)=$ $[a, b]$ with $a<b$. Let $A=\left\{x \in X: T\left(x, y_{0}\right)=a\right\}, B=\left\{x \in X: T\left(x, y_{0}\right)=b\right\}$ and $C=\left\{x \in X: a<T\left(x, y_{0}\right)<b\right\}$. The sets $A, B$ and $C$ partitioned $X$ with $A$ and $B$ being closed in $X$ and therefore compact. Since sup $f(C)<\infty$ there exists $x_{0} \in C$ such that $f\left(x_{0}\right) \geqq f(x)-\varepsilon$ for all $x \in C$.

We first let $M:=\left\{y \in Y: a<T\left(x_{0}, y\right)<b\right\}$.

Similar to the proof lines in Theorem $1, M$ is seen to be a neighbourhood of $y_{0}$ and $g(y) \leqq g\left(y_{0}\right)+\varepsilon$ for all $y \in M$.

Secondly, we let $N:=\left\{y \in Y: T(x, y)<T\left(x_{0}, y_{0}\right)<T\left(x^{\prime}, y\right)\right.$ for all $x \in A$, $\left.x^{\prime} \in B\right\}$. We proceed to show that $N$ is a neighbourhood of $y_{0}$.

For each $x \in A$ we have $\left.T\left(x, y_{0}\right)=a \in\right]-\infty, T\left(x_{0}, y_{0}\right)[. T$ is jointly continuous and so there exist neighbourhoods $U(x), V_{x}\left(y_{0}\right)$ of $x$ and $y_{0}$ respectively such that $\left.T\left(U(x), V_{x}\left(y_{0}\right)\right) \subseteq\right]-\infty, T\left(x_{0}, y_{0}\right)$ [. Now, because $A$ is compact, there exists a finite subset $A^{\prime} \subseteq A$ such that $\bigcup\left\{U(x): x \in A^{\prime}\right\} \supseteq$ $A$. The finite intersection $V:=\bigcap\left\{V_{x}\left(y_{0}\right): x \in A^{\prime}\right\}$ is then a neighbourhood of $y_{0}$ and $\left.T(A, V) \subseteq\right]-\infty, T\left(x_{0}, y_{0}\right)$ [. Similarly, there exists a 
neighbourhood $W$ of $y_{0}$ such that $\left.T(B, W) \subseteq\right] T\left(x_{0}, y_{0}\right), \infty[$. Now $N \supseteq V \cap W$ and is a neighbourhood of $y_{0}$.

For each $y \in N, T(X, y)$ is an interval of $R$. The fact that $T(A, y) \subseteq$ ] $-\infty, T\left(x_{0}, y_{0}\right)[$ and $T(B, y) \subseteq] T\left(x_{0}, y_{0}\right), \infty\left[\right.$ implies $T\left(x_{0}, y_{0}\right) \in T(C, y)$. Thus there exists $x \in C$ such that $T\left(x_{0}, y_{0}\right)=T(x, y)$. It follows that $f\left(x_{0}\right)+g\left(y_{0}\right)=f(x)+g(y)$. Since $f\left(x_{0}\right) \geqq f(x)-\varepsilon$ we have $g\left(y_{0}\right)-\varepsilon \leqq g(y)$.

$M \cap N$ is then a neighbourhood of $y_{0}$ and $g\left(y_{0}\right)-\varepsilon \leqq g(y) \leqq g\left(y_{0}\right)+\varepsilon$ for all $y \in M \cap N$. This proves the continuity of $g$ at $y_{0}$.

THEOREM 3. For equation (1), if $X$ is connected, $T$ is continuous in each variable and $f$ is nonconstant and bounded on $X$ (from both sides), then $g$ is continuous on $Y$.

Proof. Let $y_{0} \in Y$ and $\varepsilon>0$ be arbitrarily given.

The nonconstancy of $f$ in equation (1) implies that $T\left(\cdot, y_{0}\right)$ is nonconstant. $T\left(X, y_{0}\right)$ is then a nondegenerated interval of $R$ and there exist $t_{1}, t_{2} \in T\left(X, y_{0}\right)$ with $t_{1}<t_{2}$. The set $B=\left\{x \in X: T\left(x, y_{0}\right) \in\right] t_{1}, t_{2}[\}$ is mapped by $T\left(\cdot, y_{0}\right)$ onto $] t_{1}, t_{2}[$. Since $f$ is bounded from above on $B$ there exists $x_{0} \in B$ such that $f\left(x_{0}\right) \geqq f(x)-\varepsilon$ for all $x \in B$. If we set

$$
M:=\left\{y \in Y: T\left(x_{0}, y\right) \in\right] t_{1}, t_{2}[\}
$$

we see that $M$ is a neighbourhood of $y_{0}$. Furthermore for each $y \in M$, $\left.T\left(x_{0}, y\right) \in\right] t_{1}, t_{2}\left[=T\left(B, y_{0}\right)\right.$ and so there exists $x \in B$ such that $T\left(x_{0}, y\right)=$ $T\left(x, y_{0}\right)$. It follows that $f\left(x_{0}\right)+g(y)=f(x)+g\left(y_{0}\right)$. As $f\left(x_{0}\right) \geqq f(x)-\varepsilon$ we have $g(y) \leqq g\left(y_{0}\right)+\varepsilon$.

The above argument applies to the functions $\tilde{f}=-f, \tilde{g}=-g$, and $\tilde{h}=-h$ satisfying again equation (1). Hence there exists a neighbourhood $\tilde{M}$ of $y_{0}$ such that $\tilde{g}(y) \leqq \tilde{g}\left(y_{0}\right)+\varepsilon$ for all $y \in \tilde{M}$, i.e. $g\left(y_{0}\right)-\varepsilon \leqq g(y)$.

On the neighbourhood $M \cap \tilde{M}$ we have $g\left(y_{0}\right)-\varepsilon \leqq g(y) \leqq g\left(y_{0}\right)+\varepsilon$ for every $y \in M \cap \tilde{M}$. This proves the continuity of $g$.

3. Some examples. The connectedness of $X$ is a common assumption in Theorems 1, 2 and 3. Its essentiality can be seen from the following example.

EXAMPLE 1. We take $X=\{0,1\}$ the discrete space $\subseteq R, Y=R$ the reals with the usual topology, $T(x+y)=x+y, f: X \rightarrow Y$ the natural inclusion map, $g=h: R \rightarrow R$ an additive function of the reals which is continuous at no place and leaving the rationals fixed. Obviously equation (1) is satisfied, $X$ is locally connected and compact, $T$ is jointly continuous and $f$ is bounded, nonconstant on $X$, while $g$ is continuous at no place.

However, connectedness of $X$ alone is not sufficient to give Theorems 1 and 2 . This has been shown by $\mathrm{C}$. Hipp who gave the following example. 
EXample 2 (By C. Hipp). Let $X=Y=R, Y$ endowed with the cannonical topology $\tau$ on $R$ and $X$ endowed with the topology $\tau_{1}$ generated by $\tau$ and all subsets of $R$ containing the rational numbers $Q$. Then $\left(X, \tau_{1}\right)$ is connected. Let $\phi$ be a discontinuous (with respect to $\tau$ ) solution of the Cauchy equation

$$
\phi(x)+\phi(y)=\phi(x+y) \text { for all } x, y \in R .
$$

As for each $x \in X, \phi$ is bounded on $(\{x\} \cup Q) \cap(x-1, x+1)$ which is a $\tau_{1}$ neighbourhood of $x$, we have the local boundedness of $\phi$ on $\left(X, \tau_{1}\right)$. The map $T$ with $T(x, y)=x+y$ is jointly continuous on $(X, \tau) \times(Y, \tau)$ and hence continuous on $\left(X, \tau_{1}\right) \times(Y, \tau)$. However $\phi$ is continuous on $(Y, \tau)$ at no place.

The local connectedness of $X$ for Corollary 1 is by no means redundant. We illustrate this by the following example.

EXAmPle 3. We take $X=\left\{n^{-1}: n=1,2, \cdots\right\} \cup\{0\}$ as a subspace of $R, T(x, y)=x+y \sqrt{ } 2$ on $X \times X, f(x)=0$ if $x \neq 0$ and $f(0)=1, h\left(n^{-1}\right)=$ $h\left(n^{-1} \sqrt{2}\right)=1$ for all $n=1,2, \cdots$ and $h\left(n^{-1}+m^{-1} \sqrt{ } 2\right)=0$ for all $n, m=$ $1,2, \cdots$ and $h(0)=2$. Obviously, equation (2) is satisfied, $X$ fails to be locally connected at $0, T$ is jointly continuous, $f$ is bounded on $X$ but fails to be continuous at 0 .

Some uniqueness theorems concerning the continuous solutions of equations (1) and (2) are given in $\mathrm{Ng}$ [4] and Pfanzagl [5].

\section{REFERENCES}

1. J. Aczél, Lectures on functional equations and their applications, Math. in Sci. and Engineering, vol. 19, Academic Press, New York, 1966. MR 34 \#8020.

2. G. Darboux, Sur la théorème fondamental de la géométrie projective, Math. Ann. 17 (1880), 55-61.

3. J. L. Denny, Cauchy's equation and sufficient statistics on arcwise connected spaces, Ann. Math. Statist. 41 (1970), 401-411. MR 41 \#6346.

4. C. T. $\mathrm{Ng}$, On the functional equation $f(x)+\sum_{i=1}^{n} g_{i}\left(y_{i}\right)=h\left(T\left(x, y_{1}, y_{2}, \cdots, y_{n}\right)\right)$, Ann. Polon. Math. 27 (to appear).

5. J. Pfanzagl, On a functional equation related to families of exponential probability measures, Aequationes Math. 4 (1970), 139-142; Aequationes Math. 6 (1970), 120. MR 42 \#6972; MR 44 \#4426.

6. - On the functional equation $\varphi(x)+\varphi(y)=\psi(T(x, y))$, Aequationes Math. 6 (1970), 202-205.

7. R. L. Wilder, Topology of manifolds, Amer. Math. Soc. Colloq. Publ., vol. 32, Amer. Math. Soc., Providence, R.I., 1949. MR 10, 614.

Department of Applied Analysis and Computer Science, faculty of Mathematics, University of Waterloo, Waterloo, Ontario, Canada 